\title{
Does severe bleeding in HHT patients respond to intravenous bevacizumab? Review of the literature and
} case series*

\author{
T. Rosenberg ${ }^{1,2}$, A.D. Fialla ${ }^{3}$, J. Kjeldsen ${ }^{2,3}$, A.D. Kjeldsen ${ }^{1,2}$ \\ ' Department of ORL-Head \& Neck Surgery, Odense University Hospital, Denmark \\ Department of Clinical Research, University of Southern Denmark \\ ${ }^{3}$ Department of Medical Gastroenterology and Hepatology, Odense University Hospital, Denmark
}

Rhinology 57: 4, 242 - 251, 2019

https://doi.org/10.4193/Rhin18.289

* Received for publication:

November 28, 2018

Accepted: March 11, 2019

\begin{abstract}
Background: Hereditary Haemorrhagic Telangiectasia (HHT) is an autosomal dominant genetic disorder, with a wide variety of clinical manifestations due to the presence of multiple arteriovenous manifestations. Severe bleeding from the gastrointestinal (Gl) tract and/or epistaxis presents a significant problem in a subgroup of patients and systemic bevacizumab, an angiogenesis inhibitor, has been suggested to benefit these patients.
\end{abstract}

Objective: To perform a review of the literature concerning the efficacy of systemic bevacizumab in treatment of bleeding from the nose or Gl tract in patients with $\mathrm{HHT}$, including patients from our own HHT-center.

Methods: A literature review was performed using the guideline "Preferred Reporting Items for systematic Reviews and MetaAnalysis statement" (PRISMA).

Results: After careful selection, we finally analysed the results of eight case series and 33 case reports. Among 195 patients 171 (88\%) had reduced bleeding after bevacizumab.

Conclusions: Based on the literature review and data from our own case series, systemic bevacizumab is very promising as treatment for HHT patients with severe epistaxis and/or Gl-bleeding. However, care should be taken using bevacizumab, a potent angiogenesis inhibitor; long-term side effects have not been studied in this population. A randomized controlled study is warranted to support the results in HHT patients.

Key words: Hereditary haemorrhagic telangiectasia, $\mathrm{HHT}$, bevacizumab, epistaxis, GI-bleeding

\section{Introduction}

Hereditary Hemorrhagic Telangiectasia (HHT), also known as Osler-Weber-Rendu disease, is an autosomal dominantly inherited disease, with a high age-dependent penetrance, an extremely variable expression and a very heterogeneous phenotypic presentation. It is a rare condition affecting 1 in 5,000-8,000 patients ${ }^{(1)}$. The HHT diagnosis is based on diagnostic criteria established in 2000 (Table $1^{(2)}$ ). Approximately 800 Danes are expected to have HHT. HHT is caused by a mutation in one of three genes: ENG (MIM*131195; HHT1), ACVRL1 (MIM*601284; HHT2) or SMAD4 (MIM*600993JP-HHT: combination of juvenile polyposis and $\mathrm{HHT})^{(3)}$.

Clinical manifestations differ widely between HHT patients, even within family members carrying the same disease-causing mutation. The most common symptom is recurrent, spontaneous epistaxis due to telangiectatic lesions in the nasal mucosa. Usually, the disease debuts in childhood and progress with age; with ultimately $95 \%$ of the patients experiencing epistaxis. The severity of epistaxis differs between patients, ranging from nosebleeds occurring occasionally and lasting less than one minute to multiple gushing haemorrhages daily resulting in transfusion dependency ${ }^{(4-6)}$. Gastrointestinal (GI) bleeding 
is also a common symptom in HHT caused by telangiectatic lesions, present in 13-30\% of HHT patients. Similar to epistaxis, Gl bleeding tends to become more prominent with increasing age and in some patients resulting in anaemia and a need for repetitive blood transfusion ${ }^{(7-10)}$. In addition, patients may also suffer from symptoms caused by arteriovenous malformations in other organs ${ }^{(11)}$.

Current treatment of epistaxis ranges from intranasal medical treatment to local treatment in the nasal cavity with laser, nasal packing, and nasal closure in the worst afflicted patients. The main treatment for telangiectatic lesions in the Gl tract is, thermal probes or local laser treatment. Treatment of anaemia includes oral and intravenous (IV) iron substitution. In patients with severe bleeding blood transfusions is needed.

To reduce bleeding various medical systemic strategies, including oestrogen, octreotide, thalidomide, and tamoxifene have been used, without consistent effect ${ }^{(12-16)}$. Recently, a positive effect of systemic bevacizumab regarding severity of bleeding was reported in HHT patients with severe hepatic vascular malformation in a phase 2 trial ${ }^{(17)}$. Several case series and case reports have shown reduced bleeding in selected patients treated with systemic bevacizumab.

Bevacizumab is an anti-vascular endothelial growth factor (VEGF) monoclonal humanized antibody that prevents the binding of VEGF to the VEGF receptor on endothelial cells and in this way inhibits cellular proliferation and angiogenesis. In $\mathrm{HHT}$ the vascular malformations are thought to result from an imbalance in the process of angiogenesis driven by VEGF ${ }^{(18)}$ and increased plasma concentrations and tissue expression of VEGF in HHT patients have been found ${ }^{(19)}$. Thus it has been suggested that bevacizumab may be a treatment option for the vascular malformations in $\mathrm{HHT}$. Local treatment with nasal spray administered bevacizumab has been studied and no effect of this treatment was documented ${ }^{(20)}$.

Thus the aim of the present study was to estimate the effect of systemic IV bevacizumab in the treatment of bleeding from the nasal mucosa and/or the $\mathrm{Gl}$ tract due to telangietatic lesions in HHT patients by conducting a systematic literature review, and by reviewing our own case series.

\section{Materials and methods}

A literature review was performed in accordance with the guideline "Preferred Reporting Items for systematic Reviews and Meta-Analysis statement" (PRISMA) ${ }^{(21)}$. The review protocol has been registered in the PROSPERO International Prospective Register of Systematic Reviews, registration number CRD42018091601.

\section{Systematic literature search}

A PICOS (Population, Intervention, Comparison, Outcome, and
Table 1. Diagnostic criteria of HHT: The Curacao criteria (published 2000 Shovlin et al. $\left.{ }^{(2)}\right)$.

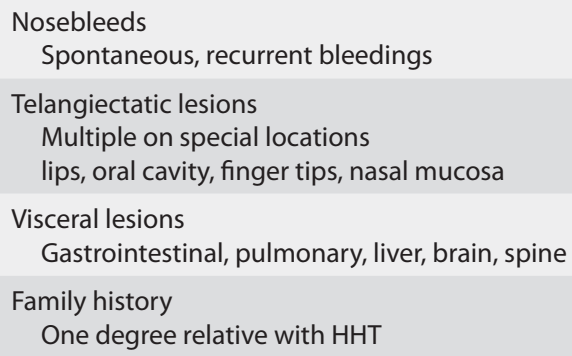

Study design) strategy ${ }^{(22)}$ was adopted for the search. The target population consisted of HHT patients suffering from epistaxis and/or Gl bleeding. The intervention was systemic IV bevacizumab, and the comparator comprised other treatment modalities prior to IV bevacizumab treatment. The outcome measures were hemoglobin levels and units of transfusion before and after treatment, and adverse events after treatment. The study design was without restrictions as long as original data were available. On January 21, 2018 we searched the electronic bibliographic databases PubMed, Embase, and Cochrane without restrictions on publication date. The search strategy was based on a combination of all available free text and Medical Subject Heading terms for $\mathrm{HHT}$, bevacizumab, and bleeding (complete search strategy can be requested from the authors). The search strategy was developed with help from a scientific librarian, and the electronic search was performed by the first author (TR) The search was repeated on September 12, 2018.

\section{Study selection}

Studies identified in the literature search were entered into EndNote version X8 (Thompson Reuters, Carlsbad, CA, USA). Duplicates were removed manually. To assess the eligibility of studies, two authors (ADF and AK) independently screened the titles and the abstracts using predefined criteria for inclusion and exclusion. Finally, the same authors independently evaluated the full text version of all publications selected after the screening process. At each step, discrepancies were solved by discussion and if needed, a third evaluator (JK) acted as arbitrator. In case of any doubt, the article proceeded to the next level. Language was restricted to English, Swedish, Norwegian, German, or Danish. Studies eligible for inclusion were randomized controlled studies, case series, and case reports.

We supplemented the review of the literature with our unpublished data on 12 patients ${ }^{(23)}$.

\section{Data item and collection process}

Two authors (ADF and AK) performed data extraction, and any discrepancies were resolved through discussion. Data were 


\begin{tabular}{|c|c|c|c|c|c|c|c|c|c|}
\hline $\begin{array}{l}\text { Patient } \\
\text { ID }\end{array}$ & $\begin{array}{l}\text { Age, } \\
\text { years }\end{array}$ & $M / F$ & HHT type & $\begin{array}{c}\text { Epistaxis } \\
\text { (y/n) at } \\
\text { treatment } \\
\text { start }\end{array}$ & $\begin{array}{l}\text { GI-bleeding } \\
\text { (y/n) at } \\
\text { treatment } \\
\text { start }\end{array}$ & $\begin{array}{l}\text { Units of } \\
\text { blood } 6 \\
\text { months } \\
\text { prior to } \\
\text { bevaci- } \\
\text { zumab }\end{array}$ & $\begin{array}{l}\text { Units of } \\
\text { blood } 6 \\
\text { months } \\
\text { after } \\
\text { induction } \\
\text { therapy }\end{array}$ & $\begin{array}{c}\text { Haemoglo- } \\
\text { bin prior } \\
\text { to bevaci- } \\
\text { zumab } \\
\text { mmol/l } \\
\text { (g/dl) }\end{array}$ & $\begin{array}{c}\text { Haemo- } \\
\text { globin } 12 \\
\text { weeks after } \\
\text { induction } \\
\text { therapy } \\
\mathrm{mmol} / \mathrm{l} \\
\text { (g/dl) }\end{array}$ \\
\hline 1 & 79 & $\mathrm{~F}$ & HHT1 & N & $Y$ & 24 & N.A.*) & $4.6(7.4)$ & $5.4(8.7)$ \\
\hline 2 & 60 & $\mathrm{~F}$ & HHT1 & N & $Y$ & 8 & 9 & $6.0(9.7)$ & $5.8(9.4)$ \\
\hline 3 & 65 & M & HНT2 & $\mathrm{N}$ & $Y$ & 7 & 0 & $7.0(11.3)$ & $10.1(16.3)$ \\
\hline 4 & 69 & M & HHT1 & $Y$ & $\mathrm{~N}$ & 6 & 0 & $5.2(8.4)$ & $7.7(12.4)$ \\
\hline 5 & 78 & $\mathrm{~F}$ & HHT2 & Y & $Y$ & 12 & 0 & $5.3(8.5)$ & $7.9(12.7)$ \\
\hline 6 & 70 & M & HHT1 & Y & $\mathrm{N}$ & 6 & 2 & $5.8(9.4)$ & $7.4(11.9)$ \\
\hline 7 & 68 & $\mathrm{~F}$ & HНT2 & $Y$ & $\mathrm{~N}$ & 0 & 0 & 7.9 (12.7) & $8,4(13.5)$ \\
\hline 8 & 60 & $\mathrm{~F}$ & HHT2 & N & $Y$ & 20 & 0 & $5.0(8.1)$ & $8.2((13.2)$ \\
\hline 9 & 68 & $\mathrm{~F}$ & HНT2 & Y & $\mathrm{N}$ & 32 & 4 & $5.9(9.5)$ & $7.9(12.7)$ \\
\hline 10 & 65 & $\mathrm{~F}$ & HHT1 & $Y$ & $\mathrm{~N}$ & 0 & 0 & $6.7(10.8)$ & $7.4(11.9)$ \\
\hline 11 & 67 & $M$ & HНT2 & $Y$ & $\mathrm{~N}$ & 11 & 0 & $5.9(9.5)$ & $8.8(14.2)$ \\
\hline 12 & 63 & $\mathrm{~F}$ & HHT2 & $Y$ & $Y$ & 0 & 0 & $5.0(8.1)$ & $7.1(11.4)$ \\
\hline Median & 68 years & & & & & 8 units & 0 units & $\begin{array}{c}5.9 \mathrm{mmol} / \mathrm{l} \\
(9.5 \mathrm{~g} / \mathrm{dl})\end{array}$ & $\begin{array}{l}7.9 \mathrm{mmol} / \mathrm{l} \\
(12.7 \mathrm{~g} / \mathrm{dl})\end{array}$ \\
\hline
\end{tabular}

*) Patient died in the follow-up period due to severe HHT manifestations.

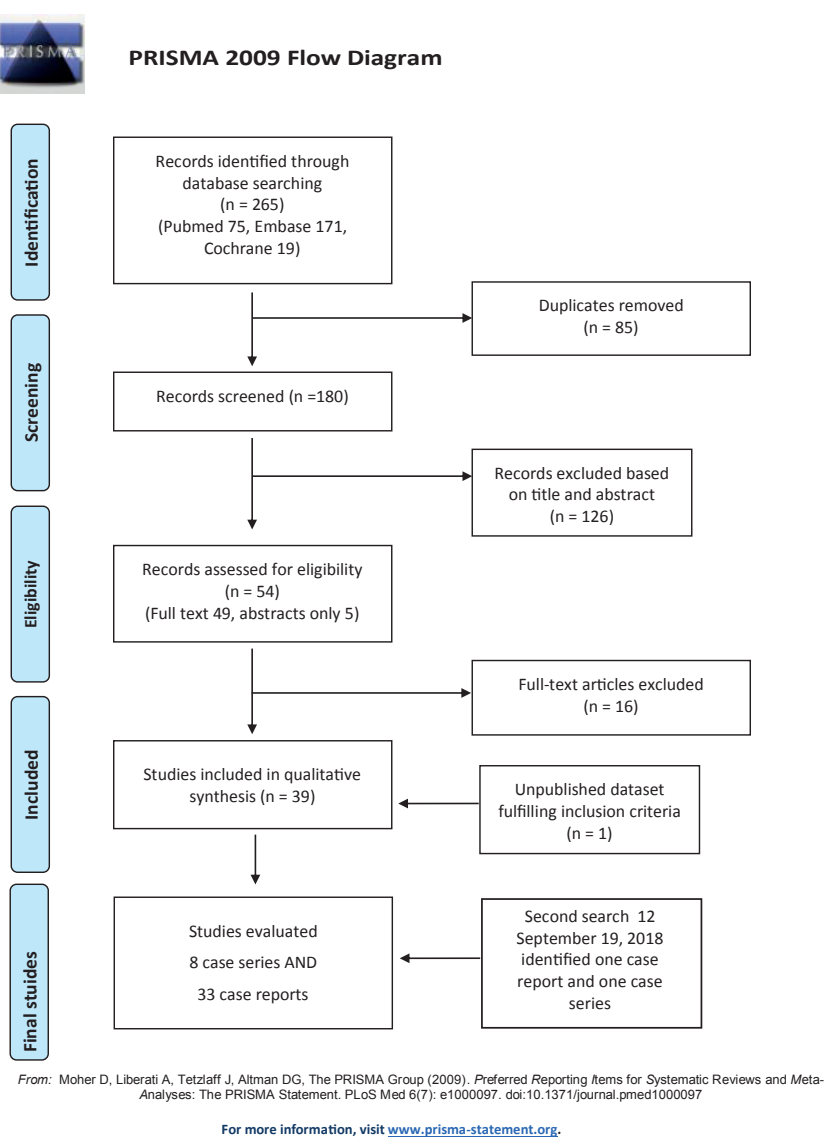

Figure 1. Study selection process. extracted with regard to the number of patients, age at bevacizumab treatment, HHT type, presence of epistaxis, GI bleeding, pulmonary arteriovenous malformations (PAVMs), hepatic AVMs (HAVMs), indication for treatment, dosage of bevacizumab, number of treatments and interval between treatments, severity of bleeding, transfusion need, hemoglobin level, and adverse events.

Patient subgroups not relevant to our study were excluded from the study population before statistical analysis.

\section{Quality assessment of individual studies}

Quality assessment of individual studies was based on the recent published guidelines "Tool for evaluating the methodological quality of case reports and case series" (24). Each study was evaluated according to four domains (selection, ascertainment, causality, and reporting) by two independent authors (AK and JK), leading to an overall assessment for each study. The evaluations were based on the research question in the present review.

\section{Synthesis of results}

A descriptive synthesis was conducted, summarizing occurrence of epistaxis and/or Gl bleeding before treatment, treatment indication, treatment efficacy, and adverse events. Data from case series and case reports were analyzed separately. 
Table 3. Study characteristics (case series).

\begin{tabular}{|c|c|c|c|c|c|c|c|c|c|c|c|}
\hline Study & n & $\begin{array}{l}\text { Gen- } \\
\text { der } \\
(\mathrm{M} / \mathrm{F})\end{array}$ & $\begin{array}{c}\text { Age } \\
\text { Median } \\
\text { (range) }\end{array}$ & $\begin{array}{l}\text { HHT- } \\
\text { type } \\
(1 / 2)\end{array}$ & Symptoms & $\begin{array}{l}\text { Treatment } \\
\text { indication }\end{array}$ & $\begin{array}{l}\text { Dose of } \\
\text { treat- } \\
\text { ment } \\
\text { (mg/ } \\
\text { kg) }\end{array}$ & $\begin{array}{l}\text { Num- } \\
\text { ber of } \\
\text { treat- } \\
\text { ments }\end{array}$ & $\begin{array}{l}\text { Interval } \\
\text { between } \\
\text { treat- } \\
\text { ments } \\
\text { (weeks) }\end{array}$ & $\begin{array}{l}\text { Effi- } \\
\text { cacy of } \\
\text { treat- } \\
\text { ment }\end{array}$ & $\begin{array}{c}\text { Adverse effects } \\
\text { (n) }\end{array}$ \\
\hline $\begin{array}{l}\text { Al-Samkari et al } \\
2018 \\
\text { Retrospective }\end{array}$ & 13 & $7 / 6$ & $\begin{array}{c}60 \\
(49-81)\end{array}$ & $2 / 3$ & $\begin{array}{l}\text { Epistaxis:11 } \\
\text { Gl-bleeding:3 } \\
\text { PAVM: } 8 \\
\text { HAVM: } 2\end{array}$ & $\begin{array}{l}\text { Epistaxis } \\
\text { Gl-bleeding }\end{array}$ & 5 & 4 & 2 & $\mathrm{DB}, \mathrm{IH}$ & $\begin{array}{l}\text { Hypertension } \\
\text { (2) }\end{array}$ \\
\hline $\begin{array}{l}\text { Chavan et al. } \\
2017 \\
\text { Prospective }\end{array}$ & 21 & $6 / 15$ & $\begin{array}{c}58 \\
(42-71)\end{array}$ & NA & $\begin{array}{l}\text { Epistaxis: } 20 \\
\text { Gl-bleeding: NA } \\
\text { PAVM: NA } \\
\text { HAVM: } 21\end{array}$ & $\begin{array}{l}\text { HAVM (high } \\
\text { cardiac output) }\end{array}$ & 5 & 6 & 2 & $\mathrm{DB}, \mathrm{IH}$ & $\begin{array}{l}\text { Hypertension } \\
(1), \text { Hemateme- } \\
\text { sis ( } 2), \text { pulmo- } \\
\text { nary embolism } \\
\text { (1) }\end{array}$ \\
\hline $\begin{array}{l}\text { Dupuis-Girod } \\
\text { et al. } 2012 \\
\text { Prospective }\end{array}$ & 25 & $1 / 24$ & $\begin{array}{c}59 \\
(35-68)\end{array}$ & $\begin{array}{l}2 / 22 \\
1 \mathrm{JP}- \\
\mathrm{HHT}\end{array}$ & $\begin{array}{l}\text { Epistaxis: } 25 \\
\text { Gl-bleeding: NA } \\
\text { PAVM: } 8 \\
\text { HAVM: } 25\end{array}$ & HAVM & 5 & 6 & 2 & DB & $\begin{array}{l}\text { Hypertension } \\
(2) \text {, headache, } \\
\text { nausea and } \\
\text { vomiting, asthe- } \\
\text { nia, abdominal } \\
\text { pain, muscular } \\
\text { pain, diarrhea, } \\
\text { rash }\end{array}$ \\
\hline $\begin{array}{l}\text { Epperla et al. } \\
2016 \\
\text { Retrospective }\end{array}$ & 5 & $5 / 0$ & $\begin{array}{c}54 \\
(43-61)\end{array}$ & NA & $\begin{array}{l}\text { Epistaxis: } 4 \\
\text { Gl-bleeding: } 1 \\
\text { PAVM: NA } \\
\text { HAVM: NA }\end{array}$ & $\begin{array}{l}\text { Epistaxis (4) } \\
\text { Gl-Bleeding (1) }\end{array}$ & 5 & 6 & 2 & DB, DT & $\begin{array}{l}\text { Hypertension } \\
\text { (2) }\end{array}$ \\
\hline $\begin{array}{l}\text { Guilhem et al. } \\
2017 \\
\text { Retrospective }\end{array}$ & 46 & $22 / 24$ & $\begin{array}{c}68 \\
(35-83)\end{array}$ & $4 / 37$ & $\begin{array}{l}\text { Epistaxis: } 44 \\
\text { Gl-bleeding: } 24 \\
\text { PAVM: } 13 \\
\text { HAVM: } 41\end{array}$ & $\begin{array}{l}\text { Epistaxis (36) } \\
\text { Gl-Bleeding } \\
(46)\end{array}$ & 5 & 6 & 2 & $\begin{array}{l}\text { Yes in } \\
74 \% \\
\text { (Not } \\
\text { speci- } \\
\text { fied) }\end{array}$ & $\begin{array}{l}\text { Infection, } \\
\text { hypertension, } \\
\text { cardiac failure, } \\
\text { wounds, ische- } \\
\text { mic events }\end{array}$ \\
\hline $\begin{array}{l}\text { lyer et al. } 2018 \\
\text { Retrospective }\end{array}$ & 34 & $13 / 21$ & $\begin{array}{c}63 \\
(57-72)\end{array}$ & $9 / 10$ & $\begin{array}{l}\text { Epistaxis: } 30 \\
\text { Gl-bleeding: } 19 \\
\text { PAVM: } 18 \\
\text { HAVM: NA }\end{array}$ & $\begin{array}{l}\text { Epistaxis (15) } \\
\text { Gl-Bleeding (4) } \\
\text { Epistaxis + Gl- } \\
\text { Bleeding (15) }\end{array}$ & 5 & 4 & 2 & $\begin{array}{l}\text { DB,IH, } \\
\text { DT }\end{array}$ & $\begin{array}{l}\text { Infusion related } \\
\text { chills, } \\
\text { Hypertension } \\
\text { (4) } \\
\text { Died during } \\
\text { follow up (3) }\end{array}$ \\
\hline $\begin{array}{l}\text { Thompson et } \\
\text { al. } 2014 \\
\text { Prospective }\end{array}$ & 6 & $3 / 3$ & $\begin{array}{c}61 \\
(46-67)\end{array}$ & NA & $\begin{array}{l}\text { Epistaxis: } 6 \\
\text { Gl-bleeding: } 0 \\
\text { PAVM: } 3 \\
\text { HAVM: NA }\end{array}$ & Epistaxis & 0.125 & 6 & 4 & DB & $\begin{array}{l}\text { Headache } \\
\text { Taste sensation }\end{array}$ \\
\hline $\begin{array}{l}\text { Own dataset } \\
\text { (unpub. 2018) } \\
\text { Retrospective }\end{array}$ & 12 & $4 / 8$ & $\begin{array}{c}67.5 \\
(60-79)\end{array}$ & $5 / 7$ & $\begin{array}{l}\text { Epistaxis: } 8 \\
\text { Gl-bleeding: } 6 \\
\text { PAVM: } 4 \\
\text { HAVM: } 1\end{array}$ & $\begin{array}{l}\text { Epistaxis + } \\
\text { Gl bleeding }\end{array}$ & 5 & 3 & 3 & IH, DT & $\begin{array}{l}\text { Hair loss (2) } \\
\text { Died during } \\
\text { follow up (1) }\end{array}$ \\
\hline Total & 162 & $\begin{array}{l}61 / \\
101\end{array}$ & & & & & & & & & \\
\hline
\end{tabular}

DB: decreased bleeding; DT: decreased transfusion; IH: increased hemoglobin

to 2017 at our institution were included. Hospital files were reviewed regarding severity of bleeding, hemoglobin levels, and transfusion need (Table 2).

\section{Results}

\section{Study selection and characteristics}

The literature search resulted in 265 records. The study selection process is illustrated in Figure 1. Forty-nine publications were analyzed as full text and five as abstracts, and their quality was assessed according to newly published recommendations ${ }^{(24)}$. Thirty-four of these publications met all eligibility criteria and were included in the review, as were five abstracts with sufficient original data and our own unpublished dataset. The repeated search in September 2018 resulted in one extra case report ${ }^{(25)}$ and one extra case series ${ }^{(26)}$. Characteristics of the eight case series ${ }^{(17,23,26-31)}$ and the 33 case reports ${ }^{(25,32-62)}$ included in the present study are summarized in Table 3 and Table 4, respectively, including study type, number of patients, gender, 
Table 4. Results of bevacizumab treatment in 33 case reports.

\begin{tabular}{|c|c|c|c|c|c|c|c|c|}
\hline \multirow[t]{2}{*}{ Study } & \multirow{2}{*}{$\begin{array}{l}\text { Sex/ } \\
\text { Age }\end{array}$} & \multirow[t]{2}{*}{ Symptoms } & \multirow{2}{*}{$\begin{array}{l}\text { Treatment } \\
\text { indication }\end{array}$} & \multicolumn{3}{|c|}{ Treatment protocol } & \multirow{2}{*}{$\begin{array}{l}\text { Efficacy of } \\
\text { treatment }\end{array}$} & \multirow[t]{2}{*}{ Adverse effects } \\
\hline & & & & $\begin{array}{c}\text { Dose } \\
(\mathbf{m g} / \mathbf{k g})\end{array}$ & No.* & $\begin{array}{l}\text { Interval } \\
\text { (weeks) }\end{array}$ & & \\
\hline Amann et al. 2015 & F 66 & $\begin{array}{l}\text { Epistaxis, } \\
\text { PAVM, HAVM }\end{array}$ & Epistaxis & 5 & 6 & 2 & DB & $\begin{array}{l}\text { Increased HR; Died during } \\
\text { follow-up after cerebral } \\
\text { abscess }\end{array}$ \\
\hline Bennesser et al. 2015 & M 66 & $\begin{array}{l}\text { Epistaxis, } \\
\text { Gl bleeding, PAVM }\end{array}$ & Epistaxis & 5 & 6 & 2 & $\begin{array}{l}\text { Yes (not } \\
\text { specified) }\end{array}$ & \\
\hline Bertoli et al. 2017 & M 47 & $\begin{array}{l}\text { Epistaxis, } \\
\text { Gl bleeding }\end{array}$ & $\begin{array}{l}\text { Epistaxis + } \\
\text { melena }\end{array}$ & 2.5 & 12 & 2 & DB, DT, IH & \\
\hline Bernardes et al 2018 & F 62 & Gl Bleeding & melena & $7.5(5)$ & $>6$ & 3 & $\mathrm{DB}, \mathrm{DT}, \mathrm{IH}$ & \\
\hline Bose et al. 2009 & M 42 & Epistaxis, PAVM & $\begin{array}{l}\text { Epistaxis + } \\
\text { hemoptysis }\end{array}$ & $10+5$ & $2+2$ & 2 & $\mathrm{DB}, \mathrm{IH}$ & \\
\hline Bourgue et al. 2012 & M 45 & Epistaxis & Epistaxis & 5 & 4 years & 4 & None & Colonic ischemia \\
\hline Brinkerhoff et al. 2011 & M 62 & Epistaxis & Epistaxis & 5 & 4 & 2 & DB, DT, IH & Treatment was repeated \\
\hline Brinkerhoff et al. 2012 & F 55 & Epistaxis & Epistaxis & $10+5$ & $2+2$ & 2 & DB, IH & $\begin{array}{l}\text { Treatment was repeated } \\
\text { once }\end{array}$ \\
\hline Busiek et al. 2010 & M 77 & $\begin{array}{l}\text { Epistaxis, } \\
\text { Gl bleeding }\end{array}$ & $\begin{array}{l}\text { Epistaxis + } \\
\text { melena }\end{array}$ & 5 & $\begin{array}{c}6 \\
\text { months }\end{array}$ & 2 & $\mathrm{DB}, \mathrm{DT}$ & \\
\hline Castillo et al. 2016 & M 67 & $\begin{array}{l}\text { Epistaxis, } \\
\text { Gl bleeding }\end{array}$ & $\begin{array}{l}\text { Epistaxis + } \\
\text { melena }\end{array}$ & 5 & 6 & 2 & DB, DT, IH & \\
\hline Epperla et al. 2015 & F 57 & $\begin{array}{l}\text { Epistaxis, } \\
\text { Gl bleeding, PAVM }\end{array}$ & $\begin{array}{l}\text { Epistaxis + } \\
\text { melena }\end{array}$ & 10 & 3 & 2 & DB, DT, IH & \\
\hline Cruikshank et al. 2011 & M 71 & $\begin{array}{l}\text { Epistaxis, } \\
\text { Gl bleeding }\end{array}$ & $\begin{array}{l}\text { Epistaxis + } \\
\text { melena }\end{array}$ & 5 & 6 & 2 & DB, DT, IH & Poor sleep \\
\hline Fleagle et al. 2012 & F 52 & $\begin{array}{l}\text { Epistaxis, } \\
\text { Gl bleeding, PAVM }\end{array}$ & $\begin{array}{l}\text { Epistaxis + } \\
\text { melena }\end{array}$ & 5 & 4 & 2 & DB, DT, IH & $\begin{array}{l}\text { Staphylococcus aureus } \\
\text { sepsis }\end{array}$ \\
\hline Flieger et al. 2006 & M 73 & Gl bleeding, HAVM & $\begin{array}{l}\text { Melena + } \\
\text { mesothe- } \\
\text { lioma }\end{array}$ & 5 & 7 & 2 & DB, DT, IH & \\
\hline Fodstad et al. 2010 & M 44 & $\begin{array}{l}\text { Epistaxis, GI blee- } \\
\text { ding, PAVM, HAVM }\end{array}$ & $\begin{array}{l}\text { Epistaxis + } \\
\text { melena }\end{array}$ & 5 & 10 & $2-3$ & DB, DT, IH & \\
\hline Follner et al. 2012 & M 82 & Gl bleeding & Melena & 5 & 3 & 2 & DB, DT, IH & \\
\hline Follner et al. 2012 & F 55 & $\begin{array}{l}\text { Epistaxis, } \\
\text { Gl bleeding }\end{array}$ & $\begin{array}{l}\text { Epistaxis + } \\
\text { melena }\end{array}$ & 5 & 3 & 2 & None & \\
\hline Huemer et al. 2016 & M 57 & Epistaxis, PAVM & Epistaxis & 1 & 16 & 2 & DB, DT, IH & $\begin{array}{l}\text { Treatment repeated after } \\
300 \text { days }\end{array}$ \\
\hline Kochanowski et al. 2015 & F 56 & $\begin{array}{l}\text { Epistaxis, } \\
\text { Gl bleeding }\end{array}$ & Melena & 3.1 & 6 & 3 & DB, DT, IH & \\
\hline Lazarki et al. 2011 & F 71 & $\begin{array}{l}\text { Epistaxis, } \\
\text { Gl bleeding, HAVM }\end{array}$ & $\begin{array}{l}\text { Epistaxis + } \\
\text { melena }\end{array}$ & 5 & 12 & 2 & DB, DT, IH & Treatment was repeated \\
\hline Lupu et al. 2013 & F 85 & Gl bleeding, HAVM & Melena & 3 & 5 & 3 & DB, DT, IH & \\
\hline Marz et al. 2011 & ?? 56 & $\begin{array}{l}\text { Epistaxis, } \\
\text { Gl bleeding }\end{array}$ & Melena & 5 & NA & 2 & DB, DT, IH & \\
\hline Marzolo et al. 2014 & F 71 & Epistaxis, HAVM & $\begin{array}{l}\text { Epistaxis } \\
+ \text { cardiac } \\
\text { failure }\end{array}$ & 5 & 6 & 3 & $\mathrm{DB}, \mathrm{DT}$, & \\
\hline Morales et al. 2012 & F 59 & Epistaxis, HAVM & $\begin{array}{l}\text { Epistaxis + } \\
\text { liver deteri- } \\
\text { oration }\end{array}$ & 5 & 6 & 2 & DB & \\
\hline Oosting et al. 2009 & M 55 & $\begin{array}{l}\text { Epistaxis, } \\
\text { pancreatitis }\end{array}$ & $\begin{array}{l}\text { Epistaxis } \\
+ \text { avm of } \\
\text { pancreas }\end{array}$ & $5+7.5$ & $10+16$ & 2 & $\mathrm{DB}$ & \\
\hline Ospina et al. 2017 & F 5 & Epistaxis, PAVM & $\begin{array}{l}\text { Epistaxis + } \\
\text { PAVM }\end{array}$ & 5 & 6 & 2 & $\mathrm{DB}$ & \\
\hline
\end{tabular}




\begin{tabular}{|c|c|c|c|c|c|c|c|c|}
\hline \multirow[t]{2}{*}{ Study } & \multirow{2}{*}{$\begin{array}{l}\text { Sex/ } \\
\text { Age }\end{array}$} & \multirow[t]{2}{*}{ Symptoms } & \multirow{2}{*}{$\begin{array}{l}\text { Treatment } \\
\text { indication }\end{array}$} & \multicolumn{3}{|c|}{ Treatment protocol } & \multirow{2}{*}{$\begin{array}{l}\text { Efficacy of } \\
\text { treatment }\end{array}$} & \multirow[t]{2}{*}{ Adverse effects } \\
\hline & & & & $\begin{array}{l}\text { Dose } \\
(\mathrm{mg} / \mathrm{kg})\end{array}$ & No.* & $\begin{array}{l}\text { Interval } \\
\text { (weeks) }\end{array}$ & & \\
\hline Ou et al. 2016 & F 58 & $\begin{array}{l}\text { Epistaxis, } \\
\text { Gl bleeding, PAVM }\end{array}$ & $\begin{array}{l}\text { Epistaxis + } \\
\text { melena }\end{array}$ & 5 & 24 & 2 & DB, DT, IH & \\
\hline Parambil et al. 2018 & M 61 & Epistaxis & Epistaxis & 5 & 6 & NA & None & \\
\hline Patrizia et al. 2010 & M 50 & $\begin{array}{l}\text { Epistaxis, } \\
\text { Gl bleeding }\end{array}$ & Epistaxis & 1 & 6 & 3 & DB, DT, IH & \\
\hline Retornaz et al. 2009 & F 65 & $\begin{array}{l}\text { Epistaxis, } \\
\text { Gl bleeding, HAVM }\end{array}$ & $\begin{array}{l}\text { Epistaxis + } \\
\text { melena }\end{array}$ & 5 & 6 & NA & DB, DT, IH & \\
\hline Riviere et al. 2011 & F 76 & Gl bleeding, PAVM & Melena & 5 & 2 & 2 & DB, DT, IH & Worsening of venous ulcers \\
\hline Sehl et al. 2015 & M 74 & $\begin{array}{l}\text { Epistaxis, } \\
\text { Gl bleeding }\end{array}$ & Melena & 3.3 & 14 & 2 & DB, DT, IH & Hypertension \\
\hline Wee et al. 2014 & M 55 & $\begin{array}{l}\text { Epistaxis, } \\
\text { Gl bleeding }\end{array}$ & Melena & 2 & 4 & 3 & DB, DT, IH & \\
\hline
\end{tabular}

PAVM: pulmonary arteriovenous malformation; HAVM: hepatic arteriovenous malformation; DB: decreased bleeding; DT: decreased transfusion; IH: increased hemoglobin *No: Number of treatments are referred as correct as possible, total numbers or numbers per months/years.

age, HHT-type, symptoms, details on treatment indication and treatment protocol, efficacy of treatment, and adverse effects.

\section{Collection process quality assessment}

An interrater agreement test was performed on abstracts giving a kappa value of 0.69 corresponding to substantial agreement between the two selecting authors. The full text reading was not assessed by interrater agreement, as the few discrepancies were solved by discussion and inclusion of a third evaluator (JK). Results of the quality assessment of the individual studies are shown in Table 5, this revealed that selection of patients were overall well performed, but that better ascertainment, causality and reporting of the treatment and treatment results, would have improved the studies.

\section{Own case series}

Twelve patients ( 8 females) with a median age of 67 years received treatment from 2013 to 2017 (Table 2). Eight patients had primarily epistaxis and four patients primarily Gl-bleeding. Eleven out of twelve patients had increase in hemoglobin level. In 7 out of 11 patients a substantial reduction in transfusion need was registered. One patient with severe manifestations of HHT with large hepatic malformation, ascites, and severe right-sided heart failure did not survive for full follow-up. The following adverse events were registered: two patients described hair-loss, one patient experienced fatigue after infusion, and one patient stopped treatment due to facial pain.

\section{Synthesis of results}

The present study included eight case series (Table 3 ) with a total of 162 patients; 101 females, 61 males. The median age ranged from 54 to 68 years. Symptoms prior to systemic IV bevaci- zumab treatment varied between studies. Eight studies included information on the prevalence of epistaxis $(n=148)$. Prevalence of $\mathrm{Gl}$ bleeding $(n=53)$ was addressed in six studies. In the study by Guilhem et al. ${ }^{(29)}$ exact numbers was difficult to extract and was estimated using the authors conclusion that $74 \%$ had benefited from the treatment.

In total, 141 of 162 patients (87\%) benefited from systemic IV bevacizumab treatment with reduced bleeding. Bleeding (epistaxis and/or Gl bleeding) was the treatment indication in 106 patients ${ }^{(23,26-30)}$, of whom 92 (87\%) had a positive effect of treatment with reduced bleeding, reduced transfusion needs, and/or increased levels of hemoglobin. Fifty-six patients from three studies $(17,29,31)$ were primarily treated with systemic IV bevacizumab due to symptomatic hepatic involvement. Forty-nine of these $(88 \%)$ also benefited from the treatment with reduced bleeding tendency.

In 32 case reports with 33 patients, 30 patients ( $91 \%$ ) benefited from treatment. The cases included 15 females, 17 males, and 1 patient without details on gender (median age was 58 years ranging from five to 85 ). All patients were treated with systemic IV bevacizumab due to bleeding (epistaxis and/or GI bleeding). A number of adverse events were reported with hypertension, occurring in 17 patients as the most common. Five patients died during follow-up (Table 3 and Table 4).

\section{Discussion}

In the present study, we aimed to estimate the effect of systemic IV bevacizumab in the treatment of bleeding from the nasal mucosa and/or the Gl tract due to teleangiectasia in patients with $\mathrm{HHT}$. We conducted a literature review and included seven published original dataset, 33 published cases, and our own unpublished dataset ${ }^{(23)}$, in total 195 HHT patients. We found 
Table 5. Quality assessment of individual studies based on "Tool for evaluating the methodological quality of case reports and case series" by Murad et al. ${ }^{(24)}$.

\begin{tabular}{|c|c|c|c|c|c|c|}
\hline Study & Country & Selection & $\begin{array}{l}\text { Ascertain- } \\
\text { ment }\end{array}$ & Causality & Reporting & Overall assessment of the study \\
\hline Al-Samkari, et al. & USA & + & + & + & + & + \\
\hline Chavan et al. & Germany & + & + & + & + & + \\
\hline Dupuis-Girod et al. & France & + & + & + & + & + \\
\hline Epperla et al. & USA & - & + & + & + & $(+)$ \\
\hline Guilhem et al. & France & - & - & + & + & (+) sampling of cases from France \\
\hline lyer et al. & USA & + & + & + & + & + \\
\hline Thompson et al. & USA & + & + & + & + & + \\
\hline Arman et al. & Austria & + & + & + & + & + \\
\hline Bennesser et al. & Marocco & + & - & - & - & $(+)$ \\
\hline Bernardes et al & Portugal & - & + & + & + & $(+)$ \\
\hline Bertoli et al. & USA & + & - & + & - & $(+)$ \\
\hline Bose et al. & USA & + & - & - & + & $(+)$ \\
\hline Bourgue et al. & USA & + & - & - & - & $(+)$ \\
\hline Brinkerhoff et al.(11) & USA & + & + & + & + & + \\
\hline Brinkerhoff et al. (12) & USA & + & + & + & + & + \\
\hline Busiek et al. & USA & + & + & - & - & $(+)$ \\
\hline Castillo et al. & Colombia & + & + & - & - & $(+)$ \\
\hline Epperla et al. & USA & + & + & + & + & + \\
\hline Cruikshank et al. & Australia & + & + & + & + & + \\
\hline Fleagle et al. & USA & + & + & + & + & + \\
\hline Flieger et al. & Germany & + & + & + & - & $(+)$ \\
\hline Fodstad et al. & Norway & + & + & + & + & + \\
\hline Follner et al. & Germany & + & - & + & - & $(+)$ two cases, one report \\
\hline Follner et al. & Germany & + & - & + & - & \\
\hline Huemer et al. & Austria & + & + & + & + & + \\
\hline Kochanowski et al. & Poland & + & - & + & + & $(+)$ \\
\hline Lazarki et al. & Greece & + & + & + & + & + \\
\hline Lupu et al. & France & + & - & + & + & $(+)$ \\
\hline Marz et al. & Germany & + & + & $(+)$ & - & $(+)$ \\
\hline Marzolo et al. & Italy & + & $(+)$ & - & - & $(+)$ \\
\hline Morales et al. & Spain & + & - & - & - & $(+)$ \\
\hline Oosting et al. & Netherlands & + & - & - & - & $(+)$ \\
\hline Ospina et al. & Colombia & + & - & - & - & $(+)$ \\
\hline Ou et al. & Canada & + & + & + & - & $(+)$ \\
\hline Parambil et al. & USA & + & - & - & - & $(+)$ \\
\hline Patrizia et al. & Italy & + & + & + & + & + \\
\hline Retornaz et al. & France & + & - & - & - & $(+)$ \\
\hline Riviere et al. & France & + & + & + & + & + \\
\hline Sehl et al. & USA & + & + & + & + & + \\
\hline Wee et al. & Korea & + & + & + & + & + \\
\hline
\end{tabular}

that $88 \%$ of the patients benefitted from the treatment with systemic IV bevacizumab. Five patients out of 195 died in the follow-up period of the reported studies. None of the deaths were directly linked to bevacizumab treatment ${ }^{(22,28,62)}$. In a newly 
published study on adverse events of bevacizumab in $\mathrm{HHT}$, there was one death out of $69 \mathrm{HHT}$ patients reported having received systemic bevacizumab. This death was suggested to be linked to bevacizumab ${ }^{(63)}$.

In this review we included all case series and case reports where intravenous bevacizumab has been used in the treatment of HHT patients, and where bleeding (epistaxis and/or Gl-bleeding) before and after treatment has been recorded. In some publications the bleeding tendency was well described, including transfusion need and hemoglobin level. In other publications the information was scarce and only included the authors opinion of whether bevacizumab reduced bleeding or not. Thus, this uncertainty about outcome is a weakness of this study. However, we felt it important to include as many cases as possible, regarding this relatively rare treatment modality.

The side effects from treatment with bevacizumab in cancer patients are well-described, and primarily include hypertension and bleeding ${ }^{(64)}$. In this study we also found that hypertension was the most common side effect.

A further weakness in the evaluation of bevacizumab is the publication bias - studies with positive results of new treatments are more likely to be published than studies with negative results.

We thoroughly searched the literature for available publications on the effect of systemic bevacizumab in the treatment of bleeding from the nasal mucosa and/or the Gl tract due to telangiectatic lesions in HHT patients. We have identified all relevant studies, but due to a limited number of original studies we found it necessary to include publications on single cases too. However, as single cases most often are highly selected this might increase the risks of publication bias. Results of the present study therefore need to be interpreted with caution. In this particular study there is also the risk that some cases may have registered twice, both as case reports from France ${ }^{(34,35,44)}$ and included in the case series ${ }^{(29)}$ from the same area.

From the present case series and case reports it is not possible to evaluate long-term effect of bevacizumab, but a majority of patients experience a clinical relevant reduction in bleeding, reducing need of transfusions, and elevating hemoglobin level. The use of systemic IV bevacizumab in the treatment of severe bleeding in HHT patients is still considered experimental and no uniform treatment protocol has been approved. However, most treatment protocols used in the present study where somewhat similar. The dose of bevacizumab administered was typically 5 $\mathrm{mg} / \mathrm{kg}$ every second or third week in up to 12 weeks (seven of eight case series and in the majority of single case reports). In one of the reported studies ${ }^{(27)}$ six patients received a small dose $(0.125 \mathrm{mg} / \mathrm{kg})$ of bevacizumab every fourth week for 24 weeks.

\section{Conclusion}

Based on the literature review and on our own case series, we believe that bevacizumab in a dose of $5 \mathrm{mg} / \mathrm{kg}$ with 3 weeks interval for 3-6 doses as induction therapy followed by maintenance therapy either at demand or at scheduled intervals is a treatment option for HHT patients with severe epistaxis and/or Gl-bleeding. However, care should be taken using bevacizumab as long-term side effects have not been studied in this population. Furthermore the treatment is contraindicated in fertile women. A randomized controlled study is warranted to support the results in HHT patients - but may be difficult due to the rarity of the disease.

\section{Acknowledgements}

None applied.

\section{Authorship contribution}

AK and JK conceived the idea, TR performed the literature search and retrieved articles, AK, JK and ADF performed the screening of the titles read and discussed the articles. All authors participated in retrieving results from the articles. TR wrote the draft of the manuscript, all authors approved the manuscript.

\section{Conflict of interest}

Non declared.

\section{References}

1. Kjeldsen AD, Vase P, Green A. Hereditary haemorrhagic telangiectasia: a populationbased study of prevalence and mortality in Danish patients. J Intern Med 1999;245:31-9.

2. Shovlin $C L$, Guttmacher $A E$, Buscarini $E$, et al. Diagnostic criteria for hereditary hemorrhagic telangiectasia (Rendu-Osler-Weber syndrome). Am J Med Genet 2000;91:66-7.

3. Torring PM, Brusgaard K, Ousager LB, Andersen PE, Kjeldsen AD. National mutation study among Danish patients with hereditary haemorrhagic telangiectasia. Clin Genet 2014;86:123-33.

4. Kjeldsen A, Aagaard KS, Torring PM, Moller S Green A. 20-year follow-up study of Danish
HHT patients-survival and causes of death Orphanet J Rare Dis 2016;11:157.

5. Kjeldsen AD, Moller TR, Brusgaard K, Vase P, Andersen PE. Clinical symptoms according to genotype amongst patients with hereditary haemorrhagic telangiectasia. J Intern Med 2005;258:349-55.

6. Hunter BN, Timmins BH, McDonald J Whitehead KJ, Ward PD, Wilson KF. An evaluation of the severity and progression of epistaxis in hereditary hemorrhagic telangiectasia 1 versus hereditary hemorrhagic telangiectasia 2. Laryngoscope 2016;126:78690.

7. Chamberlain SM, Patel J, Carter Balart J, Gossage JR, Jr., Sridhar S. Evaluation of patients with hereditary hemorrhagic telangiectasia with video capsule endoscopy: a single-center prospective study. Endoscopy 2007;39:516-20.

8. Ingrosso M, Sabba C, Pisani A, et al. Evidence of small-bowel involvement in hereditary hemorrhagic telangiectasia: a capsule-endoscopic study. Endoscopy 2004;36:1074-9.

9. Kjeldsen AD, Kjeldsen J. Gastrointestinal bleeding in patients with hereditary hemorrhagic telangiectasia. Am J Gastroenterol 2000;95:415-8.

10. Vase P, Grove O. Gastrointestinal lesions in hereditary hemorrhagic telangiectasia. Gastroenterology 1986;91:1079-83. 
11. Faughnan ME, Palda VA, Garcia-Tsao G, et al. International guidelines for the diagnosis and management of hereditary haemorrhagic telangiectasia. J Med Genet 2011;48:73-87.

12. Albinana $V$, Bernabeu-Herrero $M E$ Zarrabeitia R, Bernabeu C, Botella LM Estrogen therapy for hereditary haemorrhagic telangiectasia (HHT): Effects of raloxifene, on Endoglin and ALK1 expression in endothelial cells. Thromb Haemost 2010;103:525-34

13. Fang J, Chen X, Zhu B, et al. Thalidomide for Epistaxis in Patients with Hereditary Hemorrhagic Telangiectasia: A Preliminary Study. Otolaryngol Head Neck Surg 2017:157:217-21.

14. Hosman A, Westermann CJ, Snijder R, Disch F, Mummery $C L$, Mager JJ. Follow-up of Thalidomide treatment in patients with Hereditary Haemorrhagic Telangiectasia. Rhinology 2015;53:340-4.

15. Yaniv $E$, Preis $M$, Shevro J, Nageris B, Hadar T. Anti-estrogen therapy for hereditary hemorrhagic telangiectasia - a long-term clinical trial. Rhinology 2011;49:214-6.

16. Jameson JJ, Cave DR. Hormonal and antihormonal therapy for epistaxis in hereditary hemorrhagic telangiectasia. Laryngoscope 2004;114:705-9.

17. Dupuis-Girod S, Ginon I, Saurin JC, et al Bevacizumab in patients with hereditary hemorrhagic telangiectasia and severe hepatic vascular malformations and high cardiac output. JAMA 2012;307:948-55.

18. Han C, Choe SW, Kim YH, et al. VEGF neutralization can prevent and normalize arteriovenous malformations in an animal model for hereditary hemorrhagic telangiectasia 2. Angiogenesis 2014;17:823-30.

19. Sadick H, Naim R, Sadick M, Hormann K Riedel F. Plasma level and tissue expression of angiogenic factors in patients with hereditary hemorrhagic telangiectasia. Int J Mol Med 2005;15:591-6.

20. Stokes P, Rimmer J. Intranasal bevacizumab in the treatment of HHT -related epistaxis: a systematic review. Rhinology 2018;56:3-10.

21. Moher D, Shamseer L, Clarke M, et al Preferred reporting items for systematic review and meta-analysis protocols (PRISMA-P) 2015 statement. Syst Rev 2015;4:1.

22. Cochrane Handbook for Systematic Reviews of Interventions version 5.1.0 (updated March 2011) ed The Cochrane. The Cochrane Collaboration, 2011

23. Kjeldsen JF, A.D. Rosenberg, T. Kjeldsen, A.D Bevacizumab as Treatment of Bleeding in Patients with Hereditary Haemorrhagic Telangiectasia (HHT). Gastroenterology 2018;154:2

24. Murad MH, Sultan S, Haffar S, Bazerbach F. Methodological quality and synthesis of case series and case reports. BMJ Evid Based Med 2018:23:60-3.

25. Bernardes CS, S. Loureiro, R. Borges
V. Ramos G. Bevacizumab for Refractory Gastrointestinal Bleeding in Rendu-OslerWeber Diseas.e Portuguese Journal of Gastroenterology 2018;25:5.

26. Al-Samkari H, Kritharis A, Rodriguez-Lopez JM, Kuter DJ. Systemic bevacizumab for the treatment of chronic bleeding in hereditary haemorrhagic telangiectasia. J Intern Med 2018.

27. Thompson AB, Ross DA, Berard P, FigueroaBodine J, Livada N, Richer SL. Very low dose bevacizumab for the treatment of epistaxis in patients with hereditary hemorrhagic telangiectasia. Allergy \& rhinology (Providence, RI) 2014;5:91-5.

28. Iyer VN, Apala DR, Pannu BS, et al. Intravenous Bevacizumab for Refractory Hereditary Hemorrhagic TelangiectasiaRelated Epistaxis and Gastrointestinal Bleeding. Mayo Clin Proc 2018;93:155-66

29. Guilhem A, Fargeton $A E$, Simon $A C$, et al. Intra-venous bevacizumab in hereditary hemorrhagic telangiectasia $(\mathrm{HHT})$ : A retrospective study of 46 patients. PLoS One 2017;12:e0188943.

30. Epperla N, Kapke JT, Karafin M, Friedman $K D$, Foy P. Effect of systemic bevacizumab in severe hereditary hemorrhagic telangiectasia associated with bleeding. Am J Hematol 2016;91:E313-E4.

31. Chavan A, Schumann-Binarsch S, Schmuck $B$, et al. Emerging role of bevacizumab in management of patients with symptomatic hepatic involvement in Hereditary Hemorrhagic Telangiectasia. Am J Hematol 2017;92:E641-E4.

32. Wee JW, Jeon YW, Eun JY, Kim HJ, Bae SB, Lee KT. Hereditary hemorrhagic telangiectasia treated with low dose intravenous bevacizumab. Blood Research 2014;49:192-5.

33. Sehl ME, Gruber TM, McWilliams JP, Marder VJ. Successful management of chronic gastrointestinal hemorrhage using bevacizum$a b$ in the setting of hereditary hemorrhagic telangiectasia. Am J Hematol 2015;90:561-3.

34. Riviere S, Che H, Dupuis-Girod S, Guilpain P, Le Quellec A, Plauchu H. Trophic disorders with bevacizumab in HHT patient. Hematol Rep 2011;3:39

35. Retornaz F, Rinaldi $Y$, Duvoux C. More on bevacizumab in hereditary hemorrhagic telangiectasia [12]. N Engl J Med 2009;361:931.

36. Patrizia S, Arcangelo L, Carlo S. Low dose intravenous bevacizumab for the treatment of anaemia in hereditary haemorrhagic telangiectasia. Br J Haematol 2011;152:365-

37. Parambil JG, Woodard TD, Koc ON Pazopanib effective for bevacizumabunresponsive epistaxis in hereditary hemorrhagic telangiectasia. Laryngoscope 2018;128:2234-6.

38. Ou G, Galorport C, Enns R. Bevacizumab and gastrointestinal bleeding in hereditary hemorrhagic telangiectasia. World J Gastrointest Surg 2016:8:792-5.

39. Ospina FE, Echeverri A, Posso-Osorio I, Jaimes L, Gutierrez J, Tobon GJ. Bevacizumab as a treatment for hereditary hemorrhagic telangiectasia in children: a case report. Colomb Med (Cali) 2017:48:8893.

40. Oosting S, Nagengast W, De Vries E. More on bevacizumab in hereditary hemorrhagic telangiectasia [11]. N Engl J Med 2009;361:931.

41. Morales E, Morales R, Gutierrez E, RojasRivera J, Praga M. Raloxifene and Bevacizumab for severe complications of hereditary haemorrhagic telangiectasia in a haemodialysis patient. Clin Kidney J 2012;5:608-9.

42. Marzolo MB, E. Aggio, S. Rossetti, C. Ramazzina, S.C.A.E. . Efficacy of bevacizum$\mathrm{ab}$ in hereditary haemorrhagic telangiectasia (Rendu Osler syndrome): A case report. . Thromb Res 2014;134:2.

43. Marz W, Grauer S, Nitschke-Gerard C, et al. Successful Antiangiogenic therapy with Bevacizumab (off label) after failure of Thalidomide in a patient with hereditary hemorrhagic teleangiectasia and transfusion dependent gastrointestinal blood loss. Onkologie 2011;34:211-2.

44. Lupu A, Stefanescu C, Treton X, Attar A, Corcos O, Bouhnik Y. Bevacizumab as rescue treatment for severe recurrent gastrointestinal bleeding in hereditary hemorrhagic telangiectasia. J Clin Gastroenterol 2013;47:256-7.

45. Lazaraki G, Akriviadis E, Pilpilidis I, Parisi I, Tzilves D, Tarpangos A. Low dose of bevacizumab is safe and effective in preventing bleeding episodes in hereditary hemorrhagic telangiectasia. Am J Gastroenterol 2011;106:2204-6.

46. Kochanowski J, Sobieszczanska M, Tubek S, Zurek M, Pawelczak J. Successful therapy with bevacizumab in a case of hereditary hemorrhagic telangiectasia. Human Vaccines and Immunotherapeutics 2015;11:680-1.

47. Huemer F, Dejaco M, Grabmer C, et al. Intermittent low-dose bevacizumab in hereditary hemorrhagic telangiectasia: A case report. Wiener Klinische Wochenschrift 2017;129:141-4

48. Follner S, Ibe M, Schreiber J. Bevacizumab treatment in hereditary hemorrhagic teleangiectasia. Eur J Clin Pharmacol 2012;68:1685-6.

49. Fodstad P, Dheyauldeen S, Rinde M, Bachmann-Harildstad G. Anti-VEGF with 3-week intervals is effective on anemia in a patient with severe hereditary hemorrhagic telangiectasia. Ann Hematol 2011;90:611-2.

50. Flieger D, Hainke S, Fischbach W. Dramatic improvement in hereditary hemorrhagic telangiectasia after treatment with the vascular endothelial growth factor (VEGF) antagonist bevacizumab. Ann Hematol 2006;85:631-2.

51. Fleagle JM, Bobba RK, Kardinal CG, Freter CE. Iron deficiency anemia related to hereditary hemorrhagic telangiectasia: Response to treatment with bevacizumab. Am J Med Sci. 2012 Mar;343(3):249-51. 
52. Epperla N, Hocking W. Blessing for the bleeder: Bevacizumab in hereditary hemorrhagic telangiectasia. Clinical Medicine and Research 2015;13:32-5.

53. Cruikshank RP, Chern BW. Bevacizumab and hereditary haemorrhagic telangiectasia. Med J Aust 2011;194:324-5

54. Castillo E, Prada-Arismendy J. Bevacizumab as treatment in refractory hereditary haemorrhagic telangiectasia. Haematologica 2016;101:408.

55. Busiek D, Bernstein K. Bevacizumab rapidly and completely corrects massive transfusion requirement in a patient with hereditary hemorrhagic telangiectasia (Osler Weber Rendu Syndrome). Blood 2010;116.

56. Brinkerhoff BT, Choong NW, Treisman JS, Poetker DM. Intravenous and topical intranasal bevacizumab (Avastin) in hereditary hemorrhagic telangiectasia. American Journal of Otolaryngology - Head and Neck Medicine and Surgery 2012;33:349-51.

57. Brinkerhoff BT, Poetker DM, Choong NW. Long-term therapy with bevacizumab in hereditary hemorrhagic telangiectasia. N
Engl J Med 2011;364:688-9.

58. Bourque DA, S. Ahluwalia, J. Colonic Ulcers Secondary to Ischemia Associated with Bevacizumab Use in Hereditary Hemorrhagic Telangiectasia. Am J Gastroenterol 2012;107:2.

59. Bose P, Holter JL, Selby GB. Bevacizumab in hereditary hemorrhagic telangiectasia. N Engl J Med 2009;360:2143-4.

60. Bertoli LF, Lee PL, Lallone L, Barton JC. Intravenous Bevacizumab Therapy in a Patient with Hereditary Hemorrhagic Telangiectasia, ENG E137K, Alcoholic Cirrhosis, and Portal Hypertension. Case Rep Gastroenterol 2017;11:293-304.

61. Bennesser Alaoui H, Lehraiki M, Hamaz S, El Attar N, Fakhreddine N, Serraj K. Bevacizumab: A new success in hereditary hemorrhagic telangiectasia. Rev Med Interne 2015;36:623-5.

62. Amann A, Steiner N, Gunsilius E. Bevacizumab: an option for refractory epistaxis in hereditary haemorrhagic telangiectasia. Wiener Klinische Wochenschrift 2015;127:631-4
63. Buscarini E, Botella LM, Geisthoff $U$ et al Safety of thalidomide and bevacizumab in patients with hereditary hemorrhagic telangiectasia. Orphanet J Rare Dis. 2019 Feb 4;14(1):28

64. Keating GM. Bevacizumab: a review of its use in advanced cancer. Drugs 2014;74:1891-925.

Professor Anette Drøhse Kjeldsen,

MD, PhD

Department of Otorhinolaryngology.

Odense University Hospital

5000 Odense C

Denmark

Tel: +45 23379822

E-mail: anette.kjeldsen@rsyd.dk 